\title{
Surface Treatment of Zinc Oxide Nanoparticles by Silica Coating and Evaluation of Their Dispersibility and Photoluminescent Properties*
}

\author{
Hideki Hashimoto \\ Center for the Promotion of Project Research, Shimane University, 1060 Nishikawatsu, Matsue 690-8504, Japan \\ Ryosuke Tanino \\ Graduate School of Medical Research, Shimane University, 89-1 Enya, Izumo 693-8501, Japan
}

Michihiro Nakamura
Institute of Health Bioscience, The University of Tokushima Graduate School, 3-8-15 Kuramoto, Tokushima 770-8503, Japan

Yasuhisa Fujita ${ }^{\dagger}$

Department of Mechanical, Electrical, and Electronic Engineering,

Interdisciplinary Graduate School of Science and Engineering,

Shimane University, 1060 Nishikawatsu, Matsue 690-8504, Japan

(Received 14 January 2015; Accepted 31 October 2015; Published 21 November 2015)

\begin{abstract}
We prepared silica-coated $\mathrm{ZnO}$ nanoparticles and evaluated the dispersibility and photoluminescent properties by comparing the properties of $\mathrm{ZnO}$ nanoparticle dispersion. It was confirmed that the Zeta-potential of the nanoparticles in saline was changed from $1.52 \mathrm{mV}$ to $-43.06 \mathrm{mV}$ and the intensity of the exciton emission of $\mathrm{ZnO}$ was enhanced around 20 times by silica coating. These results indicated that the silica coating technique was effective for enhancing the dispersibility and controlling the fluorescence quenching of the emission of $\mathrm{ZnO}$.

[DOI: $10.1380 /$ ejssnt.2015.451]
\end{abstract}

Keywords: Zinc oxide; Silica; Dispersibility; Photoluminescence; Exciton emission

\section{INTRODUCTION}

Zinc Oxide $(\mathrm{ZnO})$ is a direct wide band gap $(3.37 \mathrm{eV})$ compound semiconductor and regarded as a promising ultraviolet optoelectronic material [1-3]. To achieve efficient excitonic laser action at room temperature, the binding energy of the exciton must be much higher than the thermal energy $(26 \mathrm{meV})$ [1]. In this regard, $\mathrm{ZnO}$ is a good candidate because its exciton binding energy is $\sim 60 \mathrm{meV}[1]$. Besides, $\mathrm{ZnO}$ is a safe and a low price material used as a raw material of baby powders or cosmetics $[4,5]$. Thus $\mathrm{ZnO}$ seems to be suitable for bioimaging applications. Organic dyes or Quantum dots are studied as fluorescent labeling reagents for bioimaging [6-13], but they have photobleaching behavior or toxicity for human body, hence $\mathrm{ZnO}$ is considered to be a candidate as the fluorescent labeling reagent which does not have these demerits. $\mathrm{ZnO}$ have been used as a fluorescent material using its broad (around $400 \mathrm{~nm}-600 \mathrm{~nm}$ wavelength) emissions, which are generated from oxygen vacancies $\left(V_{O}\right)$, vacant zinc $\left(\mathrm{V}_{\mathrm{Zn}}\right)$, zinc interstitials $\left(\mathrm{Zn}_{\mathrm{i}}\right)$, anti-site defects $\left(\mathrm{Zn}_{\mathrm{O}}\right)$, their ionized counterparts and complexes, and other defects in the crystalline lattices of $\mathrm{ZnO}$, which is collectively called defect emission. Although the characterization of native point defects in $\mathrm{ZnO}$ is still a question of debate [14], the band energy of the typical defect emissions were reported as follows; for example, that of $\mathrm{V}_{\mathrm{O}}$, $\mathrm{V}_{\mathrm{Zn}}$, and $\mathrm{Zn}_{\mathrm{i}}$, were around $2.5 \mathrm{eV}[15,16], 2.35 \mathrm{eV}[17,18]$,

\footnotetext{
* This paper was presented at the 7th International Symposium on Surface Science, Shimane Prefectural Convention Center $\mathrm{Ku}$ nibiki Messe), Matsue, Japan, November 2-6, 2014.

$\dagger$ Corresponding author: fujita@ecs.shimane-u.ac.jp
}

and $2.9 \mathrm{eV}$ [19], respectively. Generally the exciton emission of $\mathrm{ZnO}$ nanoparticles is decreased in solvents [3]. There are many advantages to utilize the exciton emission of $\mathrm{ZnO}$; first, the sharp emission is similar to the emission from quantum dots because the exciton emission has the atom-like energy level, second, to make relatively low price fluorescent reagents compared to quantum dots because we do not need controlling the size of $\mathrm{ZnO}$ nanoparticle with submicron order like quantum dots. Also we can use the emission wavelength around $380 \mathrm{~nm}$ which cannot be used by conventional fluorescent reagents to achieve strong fluorescence without the excitation saturation by using a fluorescent microscope with high excitation density. Therefore we are trying to apply the material as a fluorescence labeling reagent using the emission of $\mathrm{ZnO}$ exciton origin. However, $\mathrm{ZnO}$ nanoparticles are not dispersed in saline well, and the fluorescence quenching caused by energy/carrier transfer tend to be decreased the exciton emission of $\mathrm{ZnO}$ nanoparticles [20, 21]. Moreover, the toxicity of $\mathrm{ZnO}$ in vivo have been reported recently [22-25]. On the other hand, silica is known as a good insulator with large band gap energy [26], and silica-coating technique is investigated for capping toxic materials or modifying particle surface by functional group [27]. Thus we selected silica as a coating material for $\mathrm{ZnO}$ nanoparticls. There are many studies about silica-coated $\mathrm{ZnO}$ nanoparticles for bioimaging [28-32], their purpose are mainly reducing toxicity of $\mathrm{ZnO}$ or utilizing defect emissions of $\mathrm{ZnO}$ quantum dots which have more surface defects. In this study, we focused on controlling the fluorescence quenching of the exciton emission of $\mathrm{ZnO}$, prepared silica-coated $\mathrm{ZnO}$ nanoparticle dispersions and evaluated the photoluminescent properties. Meanwhile, we also evaluated the dispersibility of the prepared nanoparticles. 


\section{EXPERIMENTAL}

$\mathrm{ZnO}$ nanoparticles were prepared by using a gas evaporation method. A Zn metal (99.99\%; The NILACO, Tokyo, Japan) was melted and evaporated by the arc discharge between a carbon cathode and a $\mathrm{Zn}$ metal anode, and the $\mathrm{ZnO}$ nanoparticles were formed by the reaction of evaporated zinc and oxygen in the air [3]. The conditions of the arc discharge were the pressure of $20 \mathrm{kPa}$, the current of $50 \mathrm{~A}$, and the air flow late of $5 \mathrm{~L} / \mathrm{min}$. To prepare $\mathrm{ZnO}$ particle dispersions, $0.1 \mathrm{~g} \mathrm{ZnO}$ nanoparticles were dispersed in $20 \mathrm{~mL}$ solvent (ethanol or water) by using an ultrasonic homogenizer VCX-500 (SONICS \& MATERIALS, Newtown, CT, USA). The dispersions were centrifuged at $3000 \mathrm{~g}$ for $1 \mathrm{~min}$ at room temperature to remove large/aggregated particles. To prepare silicacoated $\mathrm{ZnO}$ particles, the $\mathrm{ZnO}$-ethanol dispersions were prepared by same way as described previously. Silicacoated $\mathrm{ZnO}$ particles were prepared by the hydrolysis and polycondensation of teraethoxysilane (TEOS) in the ZnOethanol dispersions. $\mathrm{ZnO}$ dispersion of $12.5 \mathrm{~mL}$ was put into $30 \mathrm{~mL}$ vial container with magnet stirrer, and $28 \%$ ammonia water of $0.1 \mathrm{~mL}$, ultrapure water of $0.9 \mathrm{~mL}$, and TEOS (> 95\%; KANTO CHEMICAL, Tokyo, Japan) of $1 \mathrm{~mL}$ were added into the $\mathrm{ZnO}$ dispersion, and then stirred for 24 hours at room temperature. The prepared particles were washed with ethanol and ultrapure water by centrifugation and were dispersed in ultrapure water by using an ultrasonic homogenizer. To confirm the dispersibility of the particles, the prepared particles were also dispersed in saline $(0.9 \%$ sodium chloride water; OTSUKA PHARMACEUTICAL, Tokyo, Japan) by using an ultrasonic homogenizer. In the case of observing the morphology of silica-coated $\mathrm{ZnO}$ particles, the particles-water dispersion was dried, and the dried particles were fixed on the holder for a scanning electron microscope (SEM). The morphology of prepared particles was observed by a field emission scanning electron microscope JSM-7001FA (JEOL, Tokyo, Japan). The particle size distribution of prepared particles was analyzed by using a dynamic light scattering particle size analyzer LB-550 (HORIBA, Kyoto, Japan). The Zeta-potential of prepared particles was measured by using a Zeta-potential analyzer ELSZ-1 (OTSUKA ELECTRONICS, Hirakata, Osaka, Japan). The photoluminescent properties of prepared particles were measured by using a spectorofluorometer Fluoromax 4 (HORIBA, Kyoto, Japan) with irradiating $325 \mathrm{~nm}$ light from a xenon lamp.

\section{RESULTS AND DISCUSSION}

Figure 1 is a SEM image of silica-coated $\mathrm{ZnO}$ nanoparticles. It was observed that the shape of the particles was rod-like. In the image, the blighter parts (the core part of particles) showed $\mathrm{ZnO}$ and darker parts (the shell part of particles) showed silica because $\mathrm{Zn}$ tends to emit more secondary electrons than Si by irradiating electrons. It was confirmed that the surface of $\mathrm{ZnO}$ particles was coated with silica with thickness around several tens nm. Figure 2 shows the particle size distributions for both $\mathrm{ZnO}$ particles and silica-coated $\mathrm{ZnO}$ particles in water and in

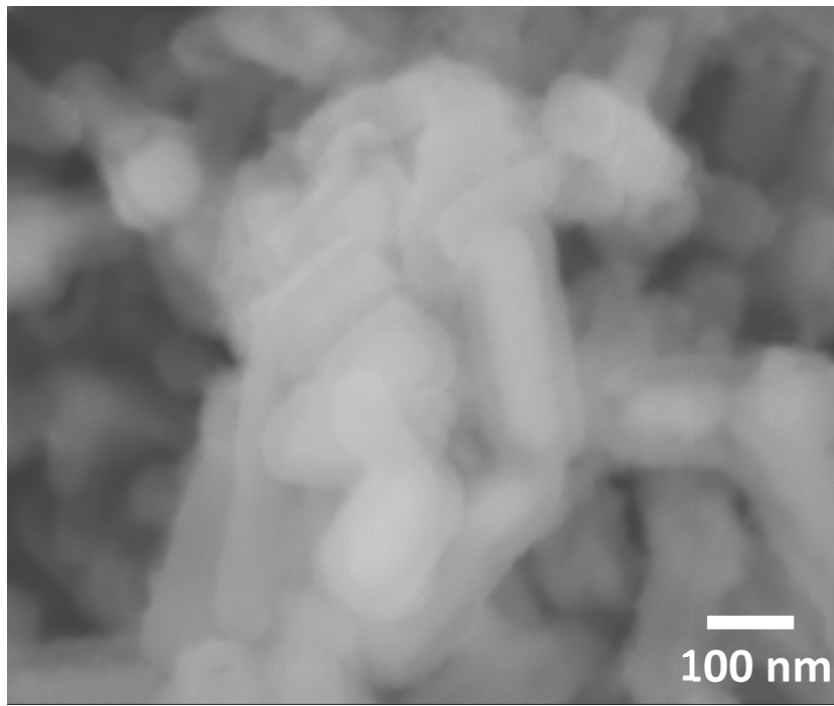

FIG. 1. SEM image of prepared silica-coated $\mathrm{ZnO}$ nanoparticles.

TABLE I. Zeta-potentials for both $\mathrm{ZnO}$ particles and silicacoated $\mathrm{ZnO}$ particles in water and in saline.

\begin{tabular}{lcc}
\hline \hline solvent & \multicolumn{2}{c}{$\begin{array}{c}\text { Zeta-potential }[\mathrm{mV}] \\
\text { Silica-coated } \mathrm{ZnO}\end{array}$} \\
\hline water & 41.30 & -46.14 \\
saline & 1.52 & -43.06 \\
\hline \hline
\end{tabular}

saline.

$\mathrm{ZnO}$ particles were dispersed better in water than in saline. On the other hand, silica-coated $\mathrm{ZnO}$ nanoparticles were dispersed well in both water and saline. Table I shows the Zeta-potential values for both $\mathrm{ZnO}$ particles and silica-coated $\mathrm{ZnO}$ particles in water and in saline. It was confirmed that $\mathrm{ZnO}$ and silica-coated $\mathrm{ZnO}$ particles showed positive and negative potentials respectively and the absolute values of Zeta-potential corresponded to the dispersibility. In water dispersion, both $\mathrm{ZnO}$ and silicacoated $\mathrm{ZnO}$ particles showed high absolute values of Zetapotential. However, in saline dispersion, the absolute value of silica-coated $\mathrm{ZnO}$ particles was high but that of $\mathrm{ZnO}$ particles was very low. These results indicated that the dispersibility of $\mathrm{ZnO}$ in saline was highly enhanced by silica coating. According to Derjaguin-Landau-VerweyOverbeck (DLVO) theory, the agglomeration and stability of particle dispersions are determined by the sum of the attractive (van der Waals) and electrostatic repulsive forces between individual particles [33, 34]. In the case of $\mathrm{ZnO}$-dispersed saline, the van der Waals force between $\mathrm{ZnO}$ particles become dominant and the agglomeration of the particles was occurred. On the other hand, in the case of silica-coated $\mathrm{ZnO}$-dispersed saline, the electrostatic repulsive force was dominant; as a result, silica-coated $\mathrm{ZnO}$ saline dispersion was stable. Figure 3 shows the photoluminescent spectra of $\mathrm{ZnO}$ dispersion and silica-coated $\mathrm{ZnO}$ dispersion. The exciton emission of $\mathrm{ZnO}$ and the defect emission were observed at around $380 \mathrm{~nm}$ and the 

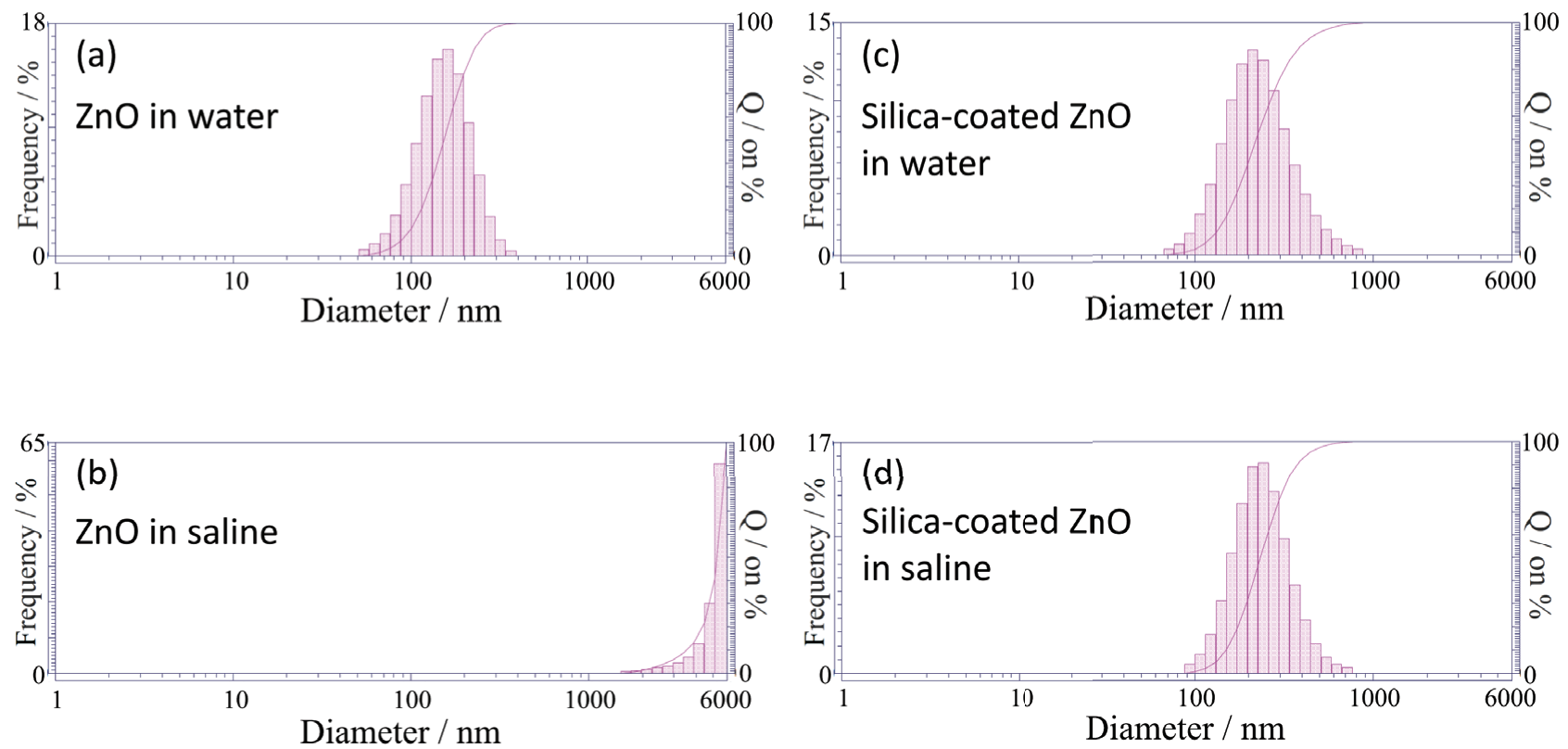

FIG. 2. Particle size distributions for both $\mathrm{ZnO}$ particles and silica-coated $\mathrm{ZnO}$ particles in water and in saline. (a) ZnO particles in water. (b) $\mathrm{ZnO}$ particles in saline. (c) Silica-coated $\mathrm{ZnO}$ particles in water. (d) Silica-coated ZnO particles in saline.

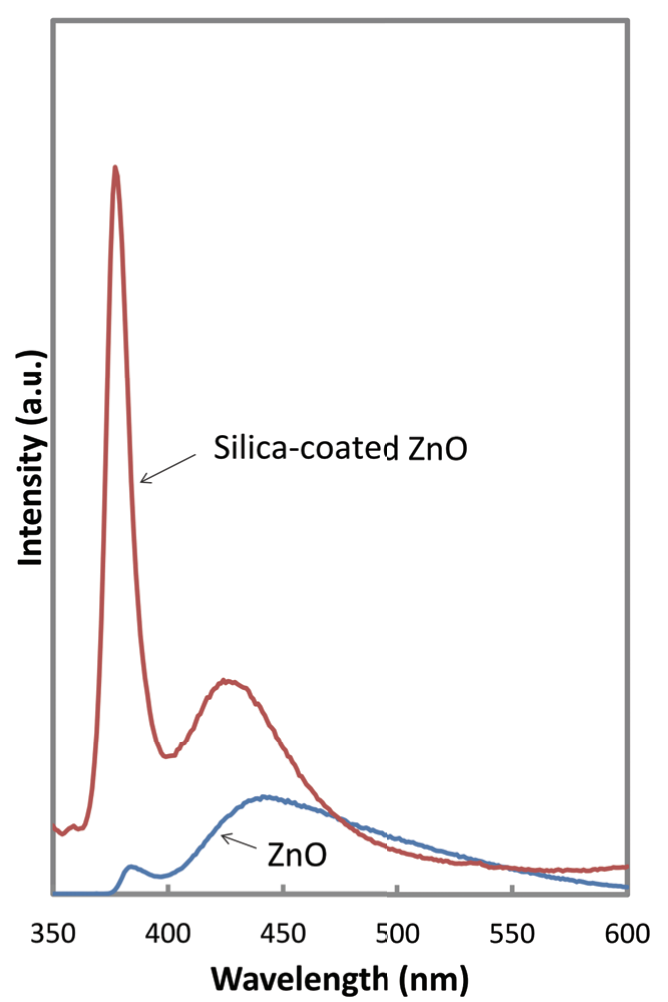

FIG. 3. Photoluminescent spectra of $\mathrm{ZnO}$ dispersion and silica-coated $\mathrm{ZnO}$ dispersion. (The wavelength of excitation light was $325 \mathrm{~nm}$.)

broad range over $400 \mathrm{~nm}$ in these spectra, respectively. It was confirmed that the intensity of the exciton emission of $\mathrm{ZnO}$ was enhanced around 20 times by silica coating. As for the exciton emission of $\mathrm{ZnO}$, it was considered that the silica layer contributed blocking the transition of the carrier energy because the band gap of silica was larger than that of $\mathrm{ZnO}$, which leading to the fluorescence quenching reduction and emission intensity enhancement. As for the defect emission of $\mathrm{ZnO}$, we also observed the enhancement of the defect emission which is not as strong as the exciton emission. Therefore the emission was mainly arisen from the donor-acceptor pair emission or the internal defects, not the surface defects. These results indicated that the carrier confinement and the surface passivation due to the core-shell structures were carried out successfully by coating $\mathrm{ZnO}$ nanoparticles with silica.

\section{CONCLUSIONS}

We had successfully prepared silica-coated $\mathrm{ZnO}$ nanoparticles. We confirmed that the dispersibility of $\mathrm{ZnO}$ in saline was enhanced by silica coating. Also we confirmed that the intensity of the exciton emission of $\mathrm{ZnO}$ was enhanced around 20 times because of the carrier confinement of $\mathrm{ZnO}$ by silica. These results suggested that the surface passivation of $\mathrm{ZnO}$ nanoparticles by silica coating is effective for enhancing the emission of $\mathrm{ZnO}$ and controlling the surface defect of $\mathrm{ZnO}$.

\section{ACKNOWLEDGMENTS}

This work was supported by the Ministry of Education, Culture, Sports, Science and Technology (MEXT) of Japan City Area Program of Shinji Lake \& Nakaumi (2009-2012). We would like to acknowledge the cooperation of the Center for Integrated Research in Science at Shimane University for providing the FE-SEM facilities which was made possible through the Tatara Project, supported by MEXT of Japan. 
[1] M. H. Huang, S. Mao, H. Feick, H. Yan, Y. Wu, H. Kind, E. Weber, R. Russo, and P. Yang, Science 292, 1897 (2001).

[2] Y. J. Xing, Z. H. Xi, Z. Q. Xue, X. D. Zhang, J. H. Song, R. M. Wang, J. Xu, Y. Song, S. L. Zhang, and D. P. Yu, Appl. Phys. Lett. 83, 1689 (2003).

[3] K. Senthilkumar, O. Senthilkumar, K. Yamauchi, M. Sato, S. Morito, T. Ohba, M. Nakamura, and Y. Fujita, Phys. Status Solidi B 246, 885 (2009).

[4] A. K. Zak, R. Razali, W. H. Majid, and M. Darroudi, Int. J. Nanomedicine 6, 1399 (2011).

[5] F. Piccino, F. Gottschalk, S. Seeger, and B. Nowack, J. Nanopart. Res. 14, 1109 (2012).

[6] X. Michalet, F. F. Pinaud, L. A. Bentolila, J. M. Tsay, S. Doose, J. J. Li, G. Sundaresan, A. M. Wu, S. S. Gambhir, and S. Weiss, Science 307, 538 (2005).

[7] S. Santra, H. Yang, P. H. Holloway, J. T. Stanley, and R. A. Mericle, J. Am. Chem. Soc. 127, 1656 (2005).

[8] H. Arya, Z. Kaul, R. Wadhwa, K. Taira, T. Hirano, and S. C. Kaul, Biochem. Biophys. Res. Commun. 329, 1173 (2005).

[9] P. Sharma, S. Brown, G. Walter, S. Santra, and B. Moudgil, Adv. Colloid Interface Sci. 123-126, 471 (2006).

[10] S. Kim, H. E. Pudavar, A. Bonoiu, and P. N. Prasad, Adv. Mater. 19, 3791 (2007).

[11] P. Yang, N. Murase, M. Suzuki, C. Hosokawa, K. Kawasaki, T. Kato, and T. Taguchi, Chem. Commum. 46, 4595 (2010).

[12] P. Yang, M. Ando, and N. Murase, Langmuir 27, 9535 (2011).

[13] C. Li and N. Murase, J. Colloid Interface Sci. 411, 82 (2013).

[14] A. F. Kohan, G. Ceder, D. Morgan, and C. G. Van de Walle, Phys. Rev. B 61, 15019 (2000).

[15] N. E. Hsu, W. K. Hung, and Y. F. Chen, J. Appl. Phys. 96, 4671 (2004).

[16] K. Vanheusden, C. H. Seager, W. L. Warren, D. R. Tallant, and J. A. Voigt, Appl. Phys. Lett. 68, 403 (1996).

[17] Y. W. Heo, D. P. Norton, and S. J. Pearton, J. Appl. Phys. 98, 073502 (2005).
[18] X. Yang, G. Du, X. Wang, J. Wang, B. Liu, Y. Zhang, D. Liu, D. Liu, H. C. Ong, and S. Yang, J. Cryst. Growth 252, 275 (2003).

[19] B. Lin, Z. Fu, and Y. Jia, Appl. Phys. Lett. 79, 943 (2001).

[20] I. Hiromitsu, T. Ikeue, K. Karino, T. Ohno, S. Tanaka, H. Shiratori, S. Morito, Y. Fujita, and M. Handa, Chem. Phys. Lett. 474, 315 (2009).

[21] I. Hiromitsu, A. Kawami, S. Tanaka, S. Morito, R. Sasai, Y. Fujita, and M. Handa, Chem. Phys. Lett. 501, 385 (2011).

[22] D. Xiong, T. Fang, L. Yu, X. Sima, and W. Zhu, Sci. Total Environ. 409, 1444 (2011).

[23] L. Gao, S. T. Yang, S. Li, Y. Meng, H. Wang, and H. Lei, J. Appl. Toxicol. 33, 1079 (2013).

[24] X. Zhao, S. Wang, Y. Wu, H. You, and L. Lv, Aquat. Toxicol. 136-137, 49 (2013).

[25] J. Fujihara, M. Tongu, H. Hashimoto, T. Yamada, K. Kimura-Kataoka, T. Yasuda, Y. Fujita, and H. Takeshita, J. Med. Invest. 62, 45 (2015).

[26] Z. A. Weinberg, G. W. Rubloff, and E. Bassous, Phys. Rev. B 19, 3107 (1979).

[27] M. Nakamura, M. Shono, and K. Ishimura, Anal. Chem. 79, 6507 (2007).

[28] K. Matsuyama, N. Ihsan, K. Irie, K. Mishima, T. Okumura, and H. Muto, J. Colloid. Interface Sci. 399, 19 (2009).

[29] N. Hagura, T. Ogi, T. Shirahama, F. Iskandar, and K. Okuyama, J. Lumin. 131, 921 (2011).

[30] S. Panigrahi, S. Bera, and D. Basak, J. Colloid. Interface Sci. 353, 30 (2011).

[31] G. A. Sotiriou, C. Watson, K. M. Murdaugh, T. H. Darrah, G. Pyrgiotakis, A. Elder, J. D. Brain, and P. Demokritou, Environ. Sci.: Nano 1, 144 (2014).

[32] M. Ramasamy, M. Das, S. S. A. An, and D. K. Yi, Int. J. Nanomedicine 9, 3707 (2014).

[33] L. S. Ott and R. G. Finke, Coord. Chem. Rev. 251, 1075 (2007).

[34] J. Jiang, G. Oberdörster, and P. Biswas, J. Nanopart. Res. 11, 77 (2009). 